\title{
On the relation between avalanche occurrence and avalanche danger level
}

\author{
Jürg Schweizer ${ }^{1}$, Christoph Mitterer ${ }^{2}$, Frank Techel ${ }^{1}$, Andreas Stoffel ${ }^{1}$, and Benjamin Reuter ${ }^{1,3}$ \\ ${ }^{1}$ WSL Institute for Snow and Avalanche Research SLF, Davos, Switzerland \\ ${ }^{2}$ Avalanche Forecasting Service Tyrol, Innsbruck, Austria \\ ${ }^{3}$ Météo France, CNRS, CNRM, Centre d'Étude de la Neige, Grenoble, France
}

Correspondence: Jürg Schweizer (schweizer@slf.ch)

Received: 21 September 2019 - Discussion started: 30 September 2019

Revised: 28 January 2020 - Accepted: 31 January 2020 - Published: 2 March 2020

\begin{abstract}
In many countries with seasonally snow-covered mountain ranges warnings are issued to alert the public about imminent avalanche danger, mostly employing an ordinal, five-level danger scale. However, as avalanche danger cannot be measured, the characterization of avalanche danger remains qualitative. The probability of avalanche occurrence in combination with the expected avalanche type and size decide on the degree of danger in a given forecast region ( $\gtrsim 100 \mathrm{~km}^{2}$ ). To describe avalanche occurrence probability, the snowpack stability and its spatial distribution need to be assessed. To quantify the relation between avalanche occurrence and avalanche danger level, we analyzed a large data set of visually observed avalanches (13918 in total) from the region of Davos (eastern Swiss Alps, $\sim 300 \mathrm{~km}^{2}$ ), all with mapped outlines, and we compared the avalanche activity to the forecast danger level on the day of occurrence (3533 danger ratings). The number of avalanches per day strongly increased with increasing danger level, confirming that not only the release probability but also the frequency of locations with a weakness in the snowpack where avalanches may initiate from increase within a region. Avalanche size did not generally increase with increasing avalanche danger level, suggesting that avalanche size may be of secondary importance compared to snowpack stability and its distribution when assessing the danger level. Moreover, the frequency of wet-snow avalanches was found to be higher than the frequency of dry-snow avalanches for a given day and danger level; also, wet-snow avalanches tended to be larger. This finding may indicate that the danger scale is not used consistently with regard to avalanche type. Even though observed avalanche occurrence and avalanche danger level are
\end{abstract}

subject to uncertainties, our findings on the characteristics of avalanche activity suggest reworking the definitions of the European avalanche danger scale. The description of the danger levels can be improved, in particular by quantifying some of the many proportional quantifiers. For instance, based on our analyses, "many avalanches", expected at danger level 4-High, means on the order of at least 10 avalanches per $100 \mathrm{~km}^{2}$. Whereas our data set is one of the most comprehensive, visually observed avalanche records are known to be inherently incomplete so that our results often refer to a lower limit and should be confirmed using other similarly comprehensive data sets.

\section{Introduction}

Avalanche forecasting was described by McClung (2002) as the prediction of snow instability in space and time relative to a given triggering level. The main sources of uncertainty in forecasting are the unknown temporal evolution and the spatial variations in instability in the snow cover. For these reasons predictability of snow avalanche occurrence is limited; it is inversely related to scale, i.e., a probability of occurrence can be given at the regional scale, but not at the scale of a single avalanche path (Schweizer, 2008). In forecasting of natural systems, in which variations may or may not be random, a distinction is often made between forecasting and prediction. In our case, prediction means precisely defining when and where an avalanche occurs. Forecasting, on the other hand, implies describing the probability of avalanche occurrence within a certain time frame and area. Given these definitions 
it is obvious that prediction is not possible - even though it would be desirable - whereas forecasting is certainly possible but inherently includes uncertainty as the forecast is probabilistic (Silver, 2012).

Even if avalanche forecasting is probabilistic and includes uncertainty, it should be grounded in clear definitions and uncertainty should not stem from ambiguous definitions but the nature of the problem. In public forecasting, i.e., issuing bulletins describing the avalanche situation, avalanche hazard is described by one of five avalanche danger levels. The danger levels (1-Low, 2-Moderate, 3-Considerable, 4-High, 5-Very High) are defined in the avalanche danger scale that was originally agreed upon by the European Avalanche Warning Services in 1993 (EAWS, 2019a; Meister, 1995). Subsequently, a very similar five-level scale was adopted in North America (Dennis and Moore, 1997), which was later revised with an emphasis on risk communication (Statham et al., 2010). In the original European danger scale, the avalanche danger levels were defined in terms of the release (or triggering) probability, the frequency and location of triggering spots, and the potential avalanche size. All three elements are supposed to be combined when assigning a danger level to a given avalanche situation. Moreover, it is assumed that all three elements increase with increasing avalanche hazard. However, the definitions for the different danger levels are short, qualitative descriptions and leave room for widely varying interpretations (Müller et al., 2016a). Not surprisingly, a recent study that looked at forecast differences across borders of contiguous forecast areas suggests that remarkable inconsistencies in the application of the danger levels exist (Techel et al., 2018). Based on a survey among forecasters, Lazar et al. (2016) also found substantial differences in assigning a single danger rating to a given scenario of avalanche conditions. These studies demonstrate that there is a lack of quantification with regard to the three key elements and their links in the avalanche danger scale.

This lack of formal underpinnings, among other reasons, motivated the development of a conceptual model of avalanche hazard in North America, which essentially formalizes the hazard assessment process (Statham et al., 2018). However, the final step on how to derive the danger level is not described. In Europe, the so-called Bavarian matrix was developed to support the decision process in forecasting. It is basically a look-up table that allows assignment of the danger level based on the probability of avalanche release and the frequency of triggering spots (Müller et al., 2016a). Avalanche size is not explicitly considered in the Bavarian matrix. Hence, recent developments in Europe were aiming at including avalanche size and harmonizing the European with the North American approach. To this end, an approach with two matrices, a so-called likelihood matrix and a danger matrix, was suggested in an attempt to merge the concepts behind the conceptual model of avalanche hazard with the Bavarian matrix (Müller et al., 2016a, b). Also, a version of the Bavarian matrix including avalanche size was suggested (EAWS, 2020).

There are few data-driven studies that link the avalanche danger level to any of the three key elements. Haegeli et al. (2012) analyzed 2 years of public avalanche forecasts with underlying hazard assessments by Avalanche Canada. They found that the maximum likelihood of triggering had the strongest impact on danger rating selection; the second most important predictor variable was the maximum expected avalanche size. More recent analyses on the relation between the components of the conceptual model of avalanche hazard and the danger ratings showed that identical avalanche scenarios were often rated differently - possibly indicating substantial inconsistencies in the forecasting process. This finding is likely due to the lack of explicitly assigning danger ratings to the various combinations in the likelihood-magnitude chart (Clark and Haegeli, 2018; Clark, 2019).

The avalanche danger levels can also be characterized with observational data related to snow instability. In the context of a verification campaign, Schweizer et al. (2003) established typical stability distributions for the danger levels 1Low, 2-Moderate and 3-Considerable based on many snow instability tests for single avalanche situations. Likewise, signs of instability such as whumpfs, shooting cracks and recent avalanching were related to the danger levels (Jamieson et al., 2009a; Schweizer, 2010). As shooting cracks were almost 10 times more frequent at level 3-Considerable (or higher) than at level 2-Moderate (or lower), they had the most predicting power in a simple classification tree. Avalanche activity was only considered as a binary variable, which does not allow insight into the avalanche characteristics at a given danger level.

Given the lack of quantitative definitions in the avalanche danger scale, our aim is to characterize avalanche activity with regard to avalanche hazard. We therefore analyzed a large data set of avalanche observations from the region of Davos (eastern Swiss Alps) and compared the avalanche activity to the avalanche danger forecast. Though both variables are subject to uncertainty, we aim at characterizing the danger levels based on frequency, type and size of avalanche occurrence.

\section{Data and methods}

We analyzed a 21-year data set of manually observed avalanche occurrences from the region of Davos, an area of about $300 \mathrm{~km}^{2}$. Large parts of the study area ranging between 1200 and $3200 \mathrm{~m}$ a.s.l. are steep mountain terrain. According to the avalanche terrain classification (CAT) by Harvey et al. (2018), about $67 \%$ of our study area is considered avalanche terrain. The snow climate in the region of Davos can be described as transitional (McClung and Schaerer, 2006). 
Data cover the winters from 1998-1999 to 2018-2019 and include 13918 individual avalanches, which were all mapped (Fig. S1 in the Supplement). Avalanches were recorded on a daily basis by SLF as well as by ski resort staff. In the more remote parts of the study area the observations are in general less consistent. Also, during the first 6 years, fewer avalanches were recorded than during the rest of the study period.

For each avalanche, we derived avalanche length $L$ and width $W$ from a rectangle of the smallest width enclosing the mapped perimeter using the "minimum boundary geometry" tool in ArcGIS. Based on these values of avalanche length and width, we assigned the avalanche size class (1 to 4) according to the Canadian size classification (McClung and Schaerer, 2006). Since avalanches of size class 5 were rare ( $L>2000 \mathrm{~m}$ and $W>300 \mathrm{~m} ; N=11$ ), we assigned those to class 4 . Moreover, 116 avalanches were too small to derive meaningful values of length and width, but were still assigned an avalanche size of 1 (hence $N=13802$ in Table 1 and Fig. 1). Table 1 describes the criteria for size classification. Also given are the resulting median length, width and area per size class for our data set.

Figure 1 shows that our size classification based on mapped outlines reproduced the exponential increase that underlies the original proposal for the size classification well (McClung and Schaerer, 1981). They suggested classifying avalanche size $S$ based on mass and proposed five classes where $S=\log M$, with $M$ the avalanche mass given in tens of metric tons. Their intention was to derive a classification that is based on destructive power, which in the end is related to volume or length. Figure 1 suggests that estimating avalanche size based on avalanche length seems indeed feasible.

In addition, the avalanche records included information on the type of triggering (natural, person, explosives/snow grooming machine, unknown) and the type of snow conditions. The snow conditions relate to the liquid water content in the starting zone (dry, wet, mixed, unknown). Dry and wet refer to dry-snow and wet-snow avalanches, respectively, whereas mixed is less well defined and typically refers to avalanches with dry-snow conditions in the starting zone but wet-snow conditions in the track or runout zone. Our records of avalanche observations cover 1358 individual days.

We calculated the avalanche activity index (AAI) for each day using the usual weights for size classes 1 to 4 , namely $0.01,0.1,1$ and 10 , respectively (Schweizer et al., 2003). Moreover, we considered the type of triggering using weights, namely 1 for natural avalanches, 0.5 for human-triggered avalanches and 0.2 for the other artificially triggered avalanches (Föhn and Schweizer, 1995). For the avalanches with an unknown trigger we assigned a weight of 0.81 since this was the weighted average of the triggering weight considering the frequency of avalanches for the three known triggering classes. In fact, almost all of the avalanches in the unknown triggering class were likely natu-

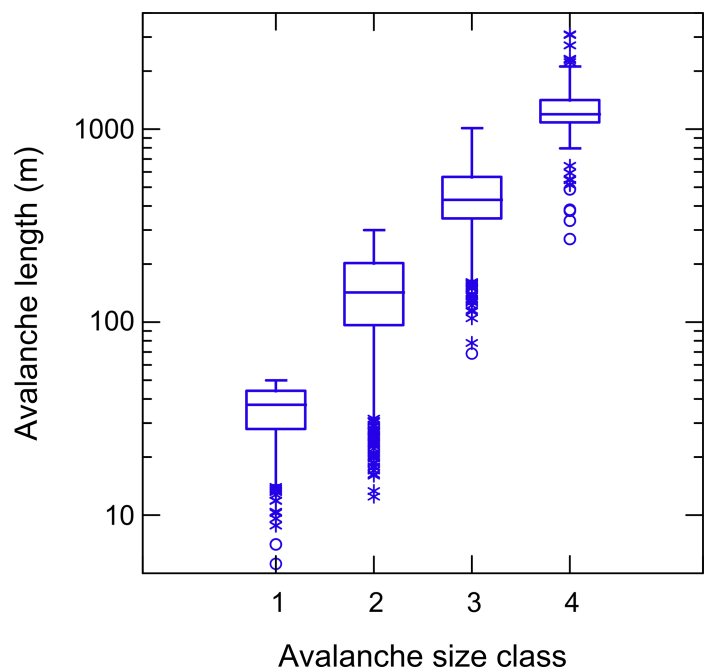

Figure 1. Distribution of avalanche length per avalanche size class for the 21-year data set of avalanche observations from the region of Davos applying the classification criteria given in Table 1. Boxes span the interquartile range from the first to third quartiles with a horizontal line showing the median. Whiskers show the range of observed values that fall within 1.5 times the interquartile range above the third and below the first quartiles. Asterisks and open circles refer to outliers and far outliers beyond the fences. Numbers indicate avalanches per class; total number of avalanches: $N=13802$.

ral avalanches so that a weight of 0.81 was appropriate. We also calculated the individual AAI for the combinations of the various types of triggering and types of snow conditions.

We then merged the data set of avalanche observations with the avalanche danger as forecast in the public bulletin for that day and the region of Davos; the forecasting region is smaller than our study area but is representative as it is located well in the study area center where most avalanches were recorded. For a total of $3533 \mathrm{~d}$ a danger rating was available. Some of the avalanches occurred outside the period when public forecasts were issued, e.g., in October or late May, and were not included for further analysis. This reduced the total number of observed avalanches to 13745 and the number of days when at least one avalanche was recorded to 1301 . This means about every third day (37\%) with a danger rating at least one avalanche was observed for the 21-year period we analyzed.

As independent data to verify the issued danger level are not available for the entire data set, we compared the avalanche activity observed on a given day to the forecast danger level on that day. Nevertheless, we did some obvious data checking. For instance, at level 4-High we expect many natural avalanches. Indeed, the two highest danger levels can be verified by avalanche activity. Therefore, we scrutinized the forecast danger levels and adjusted them to the most realistic values. All these corrections are summarized in Table S1 in the Supplement. We first checked the days with the highest danger levels, since in these cases erroneous fore- 
Table 1. Definition of avalanche size based on length and width of avalanche. Resulting median length, width and area per size class $(N=13802)$.

\begin{tabular}{lrlrrrrr}
\hline $\begin{array}{l}\text { Avalanche } \\
\text { size }\end{array}$ & $\begin{array}{r}\text { Class } \\
\text { length (m) }\end{array}$ & Operator & $\begin{array}{r}\text { Class } \\
\text { width }(\mathrm{m})\end{array}$ & $N$ & $\begin{array}{r}\text { Median } \\
\text { length }(\mathrm{m})\end{array}$ & $\begin{array}{r}\text { Median } \\
\text { width }(\mathrm{m})\end{array}$ & $\begin{array}{r}\text { Median } \\
\text { area }\left(\mathrm{m}^{2}\right)\end{array}$ \\
\hline 1: small & $<50$ & AND & $<50$ & 501 & 37 & 24 & 654 \\
2: medium & $<300$ & AND & $<300$ & 9766 & 144 & 42 & 3989 \\
3: large & {$[300,999]$} & OR & {$[300,999]$} & 3228 & 430 & 91 & 21252 \\
4: very large & $\geq 1000$ & OR & $\geq 1000$ & 307 & 1196 & 256 & 144113 \\
\hline
\end{tabular}

casts can most easily be detected: when the danger level was 4-High and no avalanches were observed, the forecast was either too high or the avalanche observations were not correctly assigned to the day of occurrence (but rather the day of observation).

We therefore started the correction process by checking the avalanche activity on the $51 \mathrm{~d}$ when the danger rating was either 4-High or 5-Very High (47 and $4 \mathrm{~d}$, respectively). We found that on 30 out of $51 \mathrm{~d}$ the avalanche activity was zero or unusually low. For each of these days, we revisited the weather, snow and avalanche conditions in the relevant period. For 23 out of the $30 \mathrm{~d}$ we down-rated the danger. For the remaining $7 \mathrm{~d}$ we corrected a temporal mismatch between the date the hazard peaked and the date avalanches were registered. For example, occasionally all avalanche observations from a $3 \mathrm{~d}$ storm had been assigned to the first or last day of the storm. For 1 out of these $7 \mathrm{~d}$ we increased the danger level from 4-High to 5-Very High. This reduced the number of days with rating 4-High from 47 to 25 and with rating 5 -Very High from 4 to 3 . Still, on $1 \mathrm{~d}$ with danger rating 4 High no avalanches were observed; this seems unlikely, but it was not possible to reconstruct the likely date of occurrence in that well-known storm period in February 1999. Unfortunately, records were in general rather inconsistent during the major storms in January and February 1999. For $1 \mathrm{~d}$ in January 2018, when the forecast danger level was 4-High and the avalanche activity very prominent $(\mathrm{AAI}=158)$ a detailed verification revealed (Bründl et al., 2019) that the forecast danger level should have been 5-Very High. This increased the total number of days with danger level 5-Very High to 4-High. Hence, after these corrections, the danger rating was 5 -Very High on $4 \mathrm{~d}$, 4-High on $25 \mathrm{~d}$ and 3-Considerable on the remaining $22 \mathrm{~d}$.

The median AAI of natural avalanches for the days with a danger rating of either 4-High or 5-Very High was 13.6, which corresponds to, for instance, only one avalanche of size 4 and a few smaller avalanches. Further quality checking revealed that there were a number of days with higher avalanche activity but lower danger level. In total on $59 \mathrm{~d}$, the avalanche danger was rated level 3-Considerable, but many natural avalanches occurred. This was also the case for $17 \mathrm{~d}$, when danger level 2-Moderate was forecast. Again, we checked all these cases against the weather, snow and avalanche conditions. For 57 of these $59 \mathrm{~d}$ we increased the rating from level 3-Considerable to 4-High since the AAI clearly indicated that the avalanche activity had been underestimated at the time of the forecast. On the remaining $2 \mathrm{~d}$ the number of natural avalanches was too low $(<10)$ to justify a change. For 12 out of $17 \mathrm{~d}$ with forecast danger level 2-Moderate, we changed the danger level to 4-High as many avalanches were observed, in most cases wet-snow avalanches, and the AAI was high. On the remaining $5 \mathrm{~d}$ we changed the danger level to 3-Considerable as the total number of natural avalanches was too low $(<10)$.

Subsequently, we considered the number of cases with level 2-Moderate danger but an avalanche activity (only naturals) higher than the median index (1.0) for days with level 3Considerable danger. There were $99 \mathrm{~d}$ with AAI $>1.0$. In 25 of these cases, the number of avalanches (size 2 and larger) was larger than 10 . For these $27 \mathrm{~d}$ we changed the danger rating to level 3-Considerable. In 19 out of these 27 cases the avalanches were wet-snow avalanches. Finally, we adjusted the danger level from level 1-Low to 3-Considerable for $2 \mathrm{~d}$, one with high natural wet-snow avalanche activity and the other with several skier-triggered avalanches.

Overall, we changed 129 out of the 3533 danger ratings $(3.7 \%)$, mostly by one danger level, occasionally by two danger levels (12\%); in most cases (106 out of 129: $82 \%$ ) we increased the danger rating since there was clearly a rather high activity of natural avalanches. In total, there were finally $94 \mathrm{~d}$ $(2.7 \%)$ with a danger rating of level 4-High, still fairly few for 21 winter seasons.

For the analyses, we stratified the data into the danger levels and compared the avalanche activity index (AAI; Schweizer et al., 2003), the proportion of avalanche sizes, the proportion of days when avalanches were observed and the average number of avalanches per day.

The number of avalanches per day relates to the probability of avalanche occurrence in our study area, i.e., one metric integrating snow stability and its distribution. The lower stability is and the more frequent the triggering locations are, the more avalanches are observed.

To compare distributions, we used the nonparametric Mann-Whitney $U$ test. We selected a level of significance $p=0.05$ to judge whether the observed differences were significant. We also checked for equality of proportions in $2 \times 2$ 
contingency tables, for instance, to decide whether the proportion of size 2 avalanches was equal for two different danger levels. Relations of continuous data such as the AAI with ordinal variables were described with the Spearman rankorder correlation coefficient $r_{\mathrm{S}}$ (Spiegel and Stephens, 1999).

\section{Results}

\subsection{Avalanche activity}

Figure 2 shows the avalanche activity index including all avalanches irrespective of triggering type or snow conditions, and Table 2 summarizes some key figures on the avalanche activity with respect to danger level. The avalanche activity, expressed as AAI, increased with increasing danger level $\left(r_{\mathrm{s}}=0.42, p<0.001\right)$. The median values were $0.15,0.21$, 1,21 and 88 for the avalanche danger levels 1-Low to 5-Very High. The increase was particularly prominent from level 3Considerable to 4-High. The highest values with AAI > 145 correspond to four distinct, well-known avalanche periods in the region of Davos: 23 April 2008, 9 March 2017, 22 January 2018 and 14 January 2019. There were only $4 \mathrm{~d}$ with danger level 5-Very High so that the corresponding AAI statistics are indicative at best.

The proportion of days when avalanches were observed at a given danger level (AAI $>0$ ) increased from about $9 \%$ at level 1-Low to $99 \%$ for level 4-High (Table 2). If only natural avalanches were considered, these proportions were $6 \%$, $16 \%, 35 \%$ and $95 \%$. At level 1-Low, natural avalanches were observed on only 1 out of $16 \mathrm{~d}$ when this danger level was forecast. At level 3-Considerable, natural avalanches were recorded every third day and at level 4-High on almost all days. This increase in the proportion of avalanche days primarily reflects that snow stability decreases with increasing danger level.

Moreover, the number of observed natural avalanches increased with increasing danger level. At the lower danger levels 1-Low and 2-Moderate, the median number of avalanches on a day with avalanche activity was 1; at level 3Considerable the median number increased to 3 , with an even stronger increase to 22 natural avalanches per day at level 4High. As the avalanche records are likely incomplete for 2 out of $4 \mathrm{~d}$ with danger level 5-Very High, the median number of natural avalanches per day was only 19 . This prominent increase in natural avalanche activity with increasing danger level is also evident in the AAI (only considering natural avalanches; median value): 0.1, 0.1, 0.2, 20 and 76 for the danger levels 1-Low to 5-Very High, respectively. The increase in the AAI or the number of avalanches per day reflects the increasing avalanche occurrence probability with increasing danger level. The prominent increase is due to decreasing snow stability and at the same time increasing frequency of locations with poor snow stability where avalanches can initiate from.

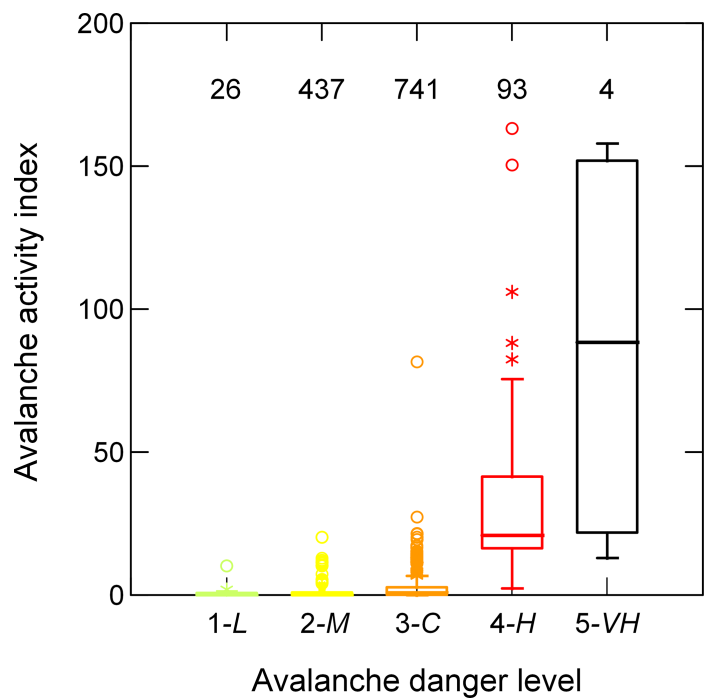

Figure 2. Avalanche activity index (AAI) per danger level (1-Low, to 5-Very High). Only days with AAI $>0$ are included. Numbers indicate number of days per danger level; total number of days: $N=$ 1301 .

Whereas the number of natural avalanches steadily increased with increasing danger level, the relation differed for the human-triggered avalanches - mainly at the higher danger levels. The proportion of days with at least one humantriggered avalanche ( $\geq$ size 2$)$ prominently increased from levels 1-Low to 3-Considerable (Table 2), about tripling from one danger level to the next; it less prominently increased to level 4-High, but was 0 at level 5-Very High - indicating that fewer people expose themselves to the hazard. The respective proportions were about $3 \%, 8 \%, 25 \%, 38 \%$ and $0 \%$.

Hence, triggering at the danger levels 1-Low and 2Moderate was rather rare. In the case a human-triggered avalanche was observed ( $\geq$ size 2 ) when the forecast avalanche danger level was 1-Low or 2-Moderate, this avalanche was in most cases (67\% and $68 \%$, respectively) the only one. In the case of natural avalanches, these proportions were lower, $47 \%$ for level 1-Low and $48 \%$ for level 2-Moderate.

The proportion of days with natural avalanches was higher than the proportion of days with human-triggered avalanches at all danger levels - even the lower ones (Table 2). The higher proportions of days with natural avalanches are primarily related to the occurrence of natural wet-snow avalanches at the lower danger levels. Below we will consider avalanche activity with regard to snow conditions and type of triggering in more detail.

\subsection{Avalanche size}

Smaller avalanches were more common than large avalanches - irrespective of the danger level (Table 3, Fig. 3a). The majority of the avalanches recorded (9649 out 
Table 2. Avalanche activity per danger level. The AAI considers all types of avalanches independent of snow conditions and trigger type; median value per day is given. Moreover, the number of days with either natural or human-triggered avalanches, at least size 2 or larger is shown.

\begin{tabular}{|c|c|c|c|c|c|c|c|c|}
\hline \multirow{2}{*}{$\begin{array}{l}\text { Danger } \\
\text { level } \\
1 \text {-Low }\end{array}$} & \multirow{2}{*}{$\begin{array}{r}\begin{array}{r}\text { Number } \\
\text { of days }\end{array} \\
303\end{array}$} & \multicolumn{2}{|c|}{$\begin{array}{c}\text { Number of days } \\
\text { with AAI }>0 \\
\text { (proportion in percent) }\end{array}$} & \multirow{2}{*}{$\begin{array}{r}\text { AAI } \\
\text { median } \\
0.15\end{array}$} & \multicolumn{2}{|c|}{$\begin{array}{c}\text { Number of days } \\
\text { with natural } \\
\text { avalanches ( } \geq \text { size } 2) \\
\text { (proportion in percent) }\end{array}$} & \multicolumn{2}{|c|}{$\begin{array}{c}\text { Number of days } \\
\text { with human-triggered } \\
\text { avalanches ( } \geq \text { size } 2 \text { ) } \\
\text { (proportion in percent) }\end{array}$} \\
\hline & & 26 & $(8.6 \%)$ & & 19 & $(6.3 \%)$ & 7 & $(2.7 \%)$ \\
\hline 2-Moderate & 1766 & 437 & $(25 \%)$ & 0.21 & 286 & $(16 \%)$ & 144 & $(8.2 \%)$ \\
\hline 3-Considerable & 1366 & 741 & $(54 \%)$ & 1.0 & 479 & $(35 \%)$ & 341 & $(25 \%)$ \\
\hline 4-High & 94 & 93 & $(99 \%)$ & 21 & 89 & $(95 \%)$ & 36 & $(38 \%)$ \\
\hline 5-Very High & 4 & 4 & $(100 \%)$ & 88 & 4 & $(100 \%)$ & 0 & $(0 \%)$ \\
\hline
\end{tabular}

of 13745) were size 2 avalanches. This size was the most frequent at all danger levels. Size 3 and size 4 avalanches were less frequent at all danger levels. In other words, for sizes 2 to 4 , the frequency of occurrence decreased with increasing avalanche size. The overall frequencies were $4 \%$, $70 \%, 23 \%$ and $2 \%$ for sizes 1 to 4 , respectively (Table 3 , bottom row).

The distributions of avalanche sizes per danger level (Fig. 3b) did not significantly differ for the danger levels 1-Low to 3-Considerable (see Table S2). For level 4-High the distribution looked similar, but still the size 2 avalanches were the most frequent followed by size 3 avalanches, but the distribution was statistically different $(U$ test, $p<0.001)$. At danger level 5-Very High the distribution was clearly different with relatively more size 4 avalanches and fewer size 2 avalanches.

Overall, about $80 \%-90 \%$ of the avalanches were size 2 or 3, whereas size 1 and size 4 avalanches were always rare (5\%), except at level 5-Very High when about $19 \%$ of the recorded avalanches were size 4 avalanches. Overall, size 2 avalanches tended to decrease, while size 3 and 4 avalanches tended to increase with increasing danger level (Fig. 3b).

The proportion of size 2 avalanches was similar (73\% to $77 \%$ ) at danger levels 1-Low to 3-Considerable (proportion test, $p>0.57$ ) but clearly decreased at danger levels 4-High $(67 \% ; p<0.001)$ and 5-Very High $(41 \% ; p<0.001)$. On the other hand, the proportion of size 3 avalanches increased from $15 \%$ at level 1-Low to $20 \%$ at both level 2-Moderate and 3-Considerable to eventually $27 \%$ at level 4-High (Table 3). The latter increase was statistically significant (proportion test, $p<0.001$ ). Size 4 avalanches were relatively most frequent at danger level 5-Very High, about 5 times more frequent than at level 4-High and about 15 times more frequent than at level 3-Considerable (Fig. 3c); the increase was statistically significant (proportion test, $p<0.001$ ).

At danger levels 1-Low and 2-Moderate avalanches were not generally smaller, but were simply less frequently observed. There was a substantial increase in avalanche occurrence with increasing danger level (Fig. 3c) as also reflected in the strong increase in the avalanche activity index (Fig. 2).
The average total number of avalanches per day was 2,3 , 8, 64 and 41 for the danger levels 1-Low to 5-Very High, respectively. Hence, in general, the frequency rather than the size increased with increasing danger level. The median avalanche length was 164, 154, 163 and $198 \mathrm{~m}$ for the danger levels 1-Low to 4-High, respectively (see Fig. S2). Hence only at danger level 4-High were the avalanches about $25 \%$ longer, and the difference was statistically significant ( $U$ test, $p<0.001)$. Avalanche length was weakly correlated with the danger levels (1-Low to 4-High) $\left(r_{\mathrm{s}}=0.13\right)$.

Whereas size 2 avalanches were clearly the most frequent at the danger levels 1-Low to 4-High and the size distributions looked partly similar, the largest avalanche observed at a given day increased with increasing danger level (Fig. 3d). At the danger levels 1-Low and 2-Moderate the largest avalanche observed was a size 2 avalanche on most days, whereas at the danger levels 4-High and 5-Very High the largest avalanches were mostly or even always size 4 avalanches, respectively.

\subsection{Snow conditions}

For about half of all avalanches (56\%) snow conditions were reported as dry, for $30 \%$ as wet and for $6.3 \%$ as mixed; for the remaining $8 \%$ (1072 avalanches) the type of avalanche snow was unknown, i.e., not recorded. As the distributions of avalanche sizes were similar for the mixed and unknown conditions ( $U$ test, $p=0.822$ ), we merged these two groups. For the three groups (dry, wet, mixed/unknown), again about $70 \%$ were size 2 avalanches and about $20 \%$ size 3 avalanches (Fig. 4a). The distributions of avalanche sizes were similar and comparable to the overall distribution (Table 3) but found to be statistically different ( $U$ test, $p<0.001$ ). Differences in size distribution with regard to snow conditions were however small. For size 2 avalanches, the proportions were even the same $(71 \%)$ for both drysnow and wet-snow conditions (proportion test, $p=0.54$ ). The proportion of wet-snow avalanches of size 3 was slightly larger $(25 \%)$ than the corresponding proportion of dry-snow avalanches $(22 \% ; p<0.001)$. Most size 4 avalanches were 
Table 3. Frequency of avalanche size per danger level (proportion in percent).

\begin{tabular}{lrrrrrr}
\hline & \multicolumn{5}{c}{$\begin{array}{c}\text { Number of avalanches } \\
\text { Avalanche size class }\end{array}$} \\
\cline { 3 - 6 } & Number of days & 1 & 2 & 3 & 4 & Total \\
\hline 1-Low & 303 & $3(5.8 \%)$ & $40(77 \%)$ & $8(15 \%)$ & $1(1.9 \%)$ & 52 \\
2-Moderate & 1766 & $73(5.5 \%)$ & $977(73 \%)$ & $262(20 \%)$ & $18(1.4 \%)$ & 1330 \\
3-Considerable & 1366 & $299(4.8 \%)$ & $4608(74 \%)$ & $1277(20 \%)$ & $82(1.3 \%)$ & 6266 \\
4-High & 94 & $220(3.7 \%)$ & $3956(67 \%)$ & $1590(27 \%)$ & $166(2.8 \%)$ & 5932 \\
5-Very High & 4 & $5(3.0 \%)$ & $68(41 \%)$ & $60(36 \%)$ & $32(19 \%)$ & 165 \\
\hline Total & 3533 & $600(4.4 \%)$ & $9649(70 \%)$ & $3197(23 \%)$ & $299(2.2 \%)$ & 13745 \\
\hline
\end{tabular}
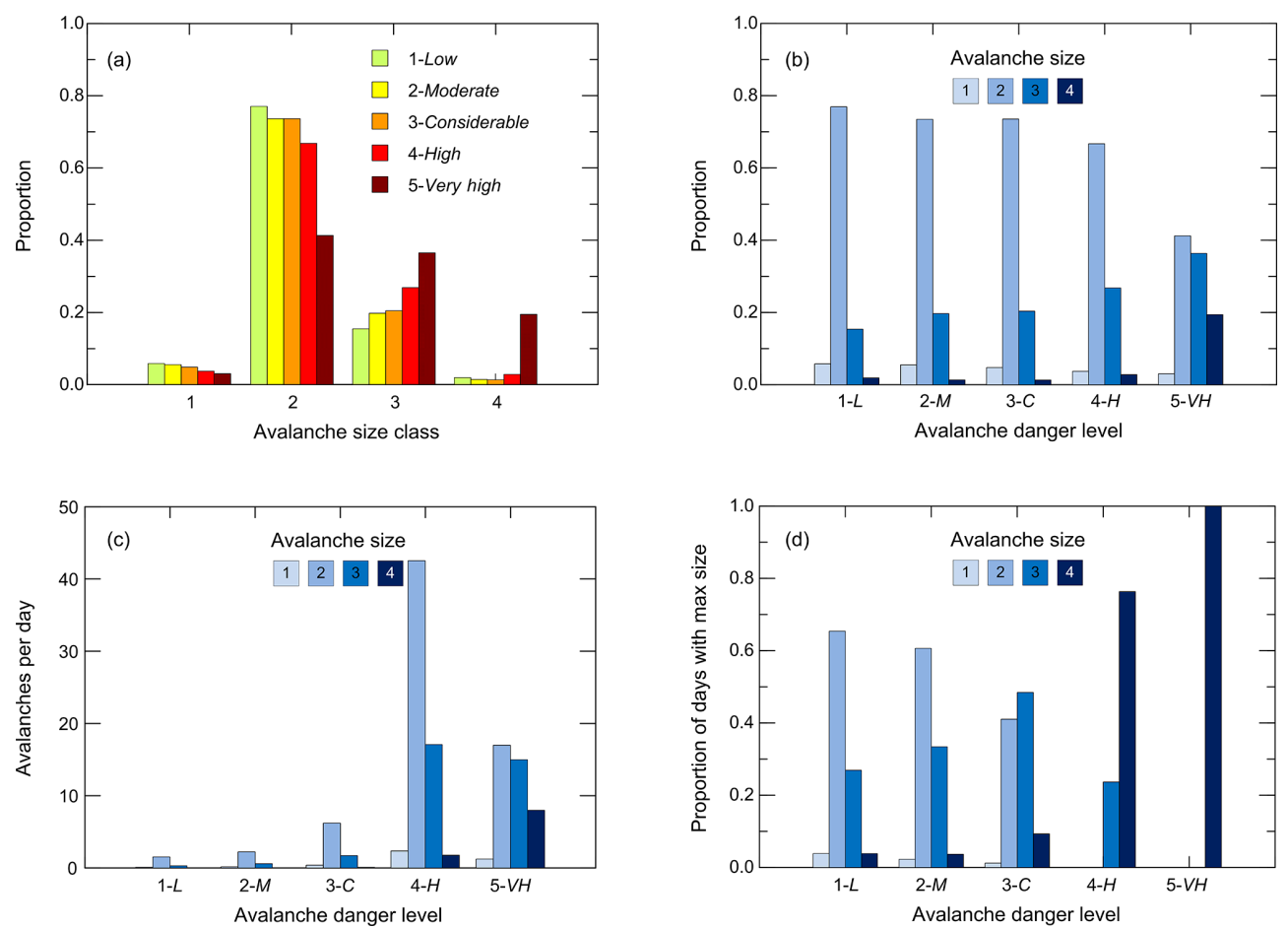

Figure 3. Avalanche size per danger level. (a) Relative frequency of avalanche sizes (1 to 4) at the danger levels 1-Low to 5-Very High. (b) Distribution of avalanche size for each danger level (same data as in Fig. 3a). (c) Average number of avalanches per day by size class for each danger level, for days with AAI $>0$. (d) Frequency of days when the largest observed avalanche was size 1, 2, 3 or 4 for each danger level. $N=13745$ avalanches on $1301 \mathrm{~d}$.

recorded for mixed or unknown conditions, relatively twice as many as for dry-snow or wet-snow conditions. In general, for mixed or unknown conditions, the avalanche size seems to be somewhat larger. Relatively fewer size 2 and more size 3 and 4 avalanches were reported. In fact, the median avalanche length was 167, 188 and $190 \mathrm{~m}$ for dry-snow, wet-snow and mixed/unknown conditions, respectively. The avalanche length distributions were similar for wet-snow and unknown/mixed conditions ( $U$ test, $p=0.55$ ), but both were different from the dry-snow conditions ( $U$ test, $p<0.001$ ). So far, in Fig. 4a, we have considered all avalanches irrespective of the type of triggering. In the following, we will only consider natural avalanches.
Whereas overall the size distributions are not very different (Fig. 4a), some differences emerge when considering the size distribution per danger level for dry-snow and wet-snow conditions (Fig. 4b, c). For instance, at level 1-Low on a day with wet-snow avalanches size 3 and size 4 avalanches were also recorded. Avalanches tended to be larger and were also more frequent (Fig. 4d). On the $10 \mathrm{~d}$ with natural wet-snow avalanches, the number of avalanches was 18 and the total AAI was 15.2. However, on the $6 \mathrm{~d}$ with natural dry-snow avalanches, the number of avalanches was eight and the total AAI was 0.71 . Hence, the AAI was on average more than 10 times larger for wet-snow than for dry-snow conditions. On the other hand, as shown in Fig. $4 d$ the average number 
of avalanches per day was 1.3 for dry-snow and 1.8 for wetsnow conditions; this difference is not particularly large. In fact, the difference in the number of avalanches per day is statistically not significant ( $U$ test, $p=0.39$ ).

At the danger levels 1-Low to 3-Considerable there were relatively more size 3 and size 4 wet-snow avalanches recorded than dry-snow avalanches. Whereas for dry-snow conditions the proportion of size 2 avalanches decreased and the proportion of size 3 avalanches increased with increasing danger level, this tendency was not evident for wet-snow conditions.

Natural avalanches under wet-snow conditions not only tended to be larger, but there were also more wet-snow avalanches than dry-snow avalanches observed on a given day. The average number of natural avalanches per day with a given danger level was larger for wet-snow than for drysnow conditions (Fig. 4d). For wet-snow avalanches the average numbers were $1.8,2.5,7.7$ and 56 for danger levels 1-Low to 4-High, for dry-snow conditions: 1.3, 2.2, 4.8 and 22. For the danger levels 3-Considerable and 4-High, the difference in the number of avalanches per day was statistically significant ( $U$ test, $p=0.001)$. Hence, the average number of avalanches per day was about 1.6 and 2.6 times larger at levels 3-Considerable and 4-High, respectively, for a day with wet-snow avalanches compared to a day with dry-snow avalanches. Overall, the 3044 natural drysnow avalanches were recorded on $482 \mathrm{~d}$ and the 3955 natural wet-snow avalanches on only 331 d; i.e., on average almost twice as many wet-snow than dry-snow avalanches per day were recorded: 12 vs. 6.3 . The difference in the number of avalanches per day between dry- and wet-snow conditions was statistically significant ( $U$ test, $p=0.001$ ) Hence, under wet-snow conditions, avalanches were not only larger, but also more frequent compared to dry-snow conditions.

\subsection{Type of triggering}

Whereas above we have compared avalanche activity with regard to snow conditions and primarily focused on natural releases, in the following we will only consider dry-snow avalanches and focus on the type of triggering, i.e., compare natural to human-triggered avalanches. For dry-snow conditions, there were about 3 times more natural $(N=3044)$ than human-triggered avalanches $(N=1036)$. For both natural and human-triggered avalanches, size 2 avalanches were most frequently observed, in $70 \%$ and $74 \%$, respectively (Fig. 5a), followed again by size 3 avalanches (24\% and $15 \%)$. Hence, the frequency distribution was again similar but statistically significantly different ( $U$ test, $p<0.001$ ). There were relatively more human-triggered avalanches of size 1 and 2, yet more natural avalanches of size 3 and 4 . For instance, size 4 avalanches were 1.7 times more frequent among the natural than the human-triggered avalanches. Still, there were 17 human-triggered size 4 avalanches recorded. In summary, natural dry-snow avalanches tended to be larger
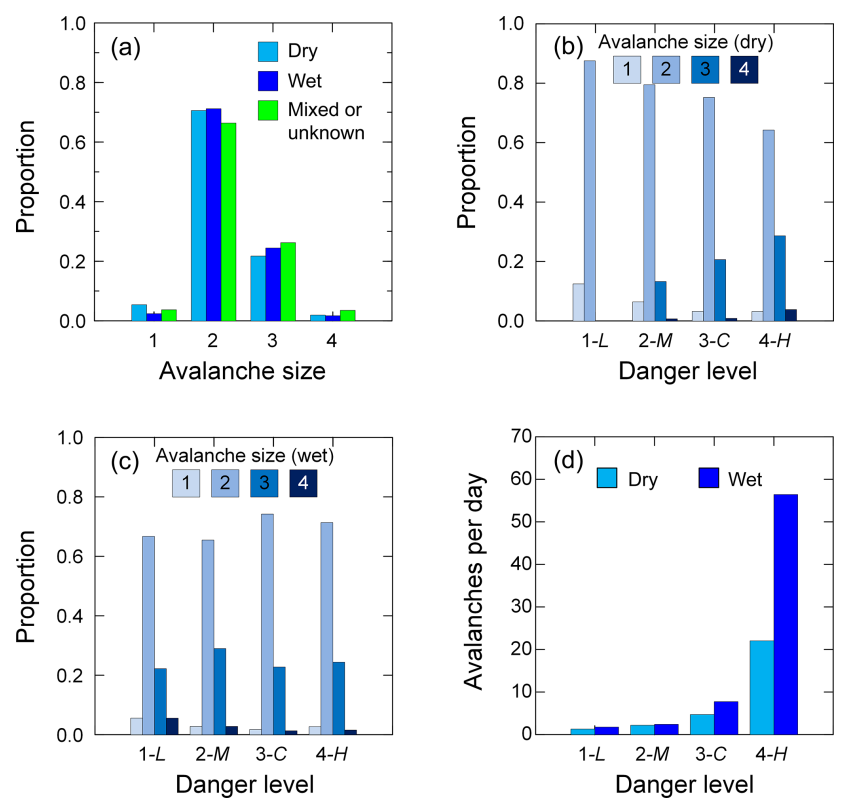

Figure 4. (a) Avalanche size distribution for dry-snow avalanches $(N=7748)$, wet-snow avalanches $(N=4056)$ and avalanches where the snow type was either recorded as mixed or it was not recorded at all $(N=1941)$. Avalanche size distribution per danger level (1-Low to 4-High) for (b) natural dry-snow avalanches $(N=$ $3044)$ and (c) natural wet-snow avalanches $(N=3955)$. (d) Number of avalanches on a day with corresponding avalanche activity per danger level for dry-snow and wet-snow conditions.

than human-triggered dry-snow avalanches. In total, $27 \%$ of the dry-snow natural avalanches were size 3 and size 4 avalanches, whereas the corresponding proportion was only $17 \%$ among the human-triggered avalanches (proportion test, $p<0.001$ ).

For the dry-snow human-triggered avalanches the size distributions at the four danger levels 1-Low to 4-High were similar to the overall distribution shown in Fig. 5a. For instance, at danger levels 3-Considerable and 4-High, the proportion of size 3 avalanches was 0.74 and 0.73 (proportion test, $p=0.77)$. For size 4 avalanches the corresponding proportions were 0.14 and 0.19 (proportion test, $p=0.36$ ). Moreover, the median avalanche length was 154, 139, 128 and $142 \mathrm{~m}$ at the danger levels 1-Low to 4-High. The corresponding length distributions per danger level were statistically not different (pairwise $U$ test, $p>0.19$ ). Hence, avalanche size did not increase with increasing danger level $\left(r_{\mathrm{s}}=0.004\right)$.

We then considered the relative frequency of avalanche days per danger level (Fig. 5b). Human-triggered as well as natural dry-snow avalanches were rare when the danger was rated as level 1-Low. Only in 6 out of $303 \mathrm{~d}(2 \%)$ with level 1-Low was a human-triggered avalanche recorded, and on $6 \mathrm{~d}$ a natural avalanche was recorded (considering all size classes). In total, there were six human-triggered and 

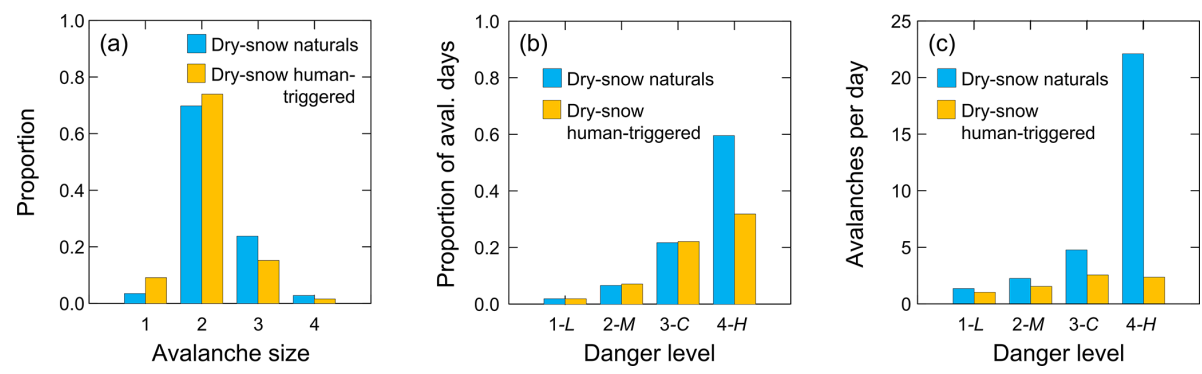

Figure 5. Type of triggering for dry-snow avalanches. (a) Avalanche size distribution for natural and human-triggered avalanches. (b) Proportion of days with either natural or human-triggered avalanches with regard to danger level. (c) Number of avalanches per day vs. danger level.

eight natural avalanches on 11 individual days; i.e., typically there was one avalanche per day when there were dry-snow avalanches at all at level 1-Low. Hence, avalanche occurrence at level 1-Low is unlikely, irrespective of the type of triggering. The proportion of days with human-triggered dry-snow avalanches increased to $7.2 \%, 22 \%$ and $32 \%$ for days with a forecast danger level of 2-Moderate to 4-High, respectively. For natural dry-snow avalanches, the corresponding percentage values were $2 \%, 6.7 \%, 22 \%$ and $60 \%$.

Not only the proportion of avalanche days but also the average number of avalanches per day prominently increased with increasing danger level (Fig. 5c). For the humantriggered avalanches at level 2-Moderate, the average number per day, when at least one avalanche was recorded, was 1.5. At level 3-Considerable it was 2.5, but at level 4High it slightly decreased to 2.3. For the natural dry-snow avalanches, which are more closely related to the release probability and do not depend on the presence of people, the increase was more prominent: 1.3, 2.2, 4.8 and 22 natural avalanches per day with danger rating 1-Low to 4-High, respectively. This corresponds to about a factor of 2, 4 and 17 increase from level 1-Low to the higher levels.

If not only dry-snow natural avalanches are considered, the increase in the average number of avalanches per day is even more prominent. Considering all natural avalanches irrespective of the snow conditions (i.e., dry, wet, mixed and unknown), the average number of avalanches per day was 2.1, 2.5, 6.0, 48 and 33 for the danger levels 1-Low to 5-Very High, respectively. The higher number of natural avalanches per day, when considering all types of snow conditions instead of dry-snow only, reflects the finding that generally more wet-snow than dry-snow avalanches were observed at a given danger level as shown above in Fig. 4d.

\section{Discussion}

We analyzed a data set of visually observed avalanches from the region of Davos (Switzerland). Even though the data set was collected with the aim to record all (or at least as many as possible) avalanches in the region of Davos, our data set certainly cannot provide the complete picture of avalanche activity, too. Small avalanches may be underreported in general. Moreover, avalanche records based on visual observations are known to be biased since during times of poor visibility it is often difficult, and sometimes even impossible, to accurately outline the avalanche extent or record the release date (van Herwijnen and Schweizer, 2011). Only with remote avalanche detection systems can the observation bias during storms be overcome, at least with regard to the temporal resolution (Lacroix et al., 2012; Ulivieri et al., 2011). A good spatial resolution can only be achieved with remote sensing from satellites (Eckerstorfer et al., 2017). However, presently the temporal resolution over the Alps is still too poor for operational purposes, since only every $6 \mathrm{~d}$ are images acquired from the same orbit, which is necessary for change detection. Hence, when we provide the number of avalanches per day observed in our study region, this number should be considered as a lower limit, since with visual observation a full coverage of the study area cannot be achieved.

Moreover, there may be other biases as it is, for instance, easier to record wet-snow than dry-snow avalanches. Also, the level of reporting varied during the 21 winter seasons. There were relatively more observations in the period of 2004-2005 to 2018-2019 than in the first 6 years of our study period. Still, size 2 and size 3 avalanches were the most frequent ones in both periods. The proportion of size 2 avalanches was about $70 \%$ in both periods (proportion test, $p=0.93$ ). The proportion of size 3 avalanches was $22 \%$ and $23 \%$ during the first and second periods, respectively, again not significantly different $(p=0.28)$. Hence, the key characteristics did not change much.

With 13918 avalanches the data set is extensive and covers many different avalanche situations - small as well as large avalanches, single avalanches as well as records from intense storms with many avalanches - in short, it seems a rather complete data set. A much smaller data set also with mapped avalanche perimeters for the surroundings of the village Zuoz in the lower Engadine (Swiss Alps) was analyzed by Stoffel et al. (1998). In France, the "Enquête permanente sur les avalanches" (EPA) is an extensive data set including the avalanche events in approximately $5000 \mathrm{ma}-$ 
jor paths in the French Alps and the Pyrenees (e.g., Eckert et al., 2010). Primarily large avalanches that can threaten infrastructure are recorded. Hence this data set seems biased towards very large avalanches and less suited for our purpose. Other extensive data sets were recorded along mountain passes, e.g., along the Milford road in New Zealand (Hendrikx et al., 2005). They found a significant lack of smaller sizes in their size class distribution, which contained 1842 avalanches. Since only larger avalanches $($ size $\geq 2.5$ ) are relevant for road safety, smaller avalanches were underreported. Similar underestimation of size 1 and size 2 avalanches was present in avalanche records observed along the highway crossing Rogers Pass (Canada) (McClung and Schaerer, 1981). Hence, our data set seems to be one of the most comprehensive ones - providing unique insight into avalanche activity. In general, the type of recording and the potential impact (e.g., whether the avalanche hit the road and/or caused damage) may represent the most relevant biases in avalanche data sets.

The avalanche activity as described based on our data set also depends on terrain characteristics and snow climate. Hence, the characteristics of avalanche activity in our study area cannot simply be transferred to other mountain regions. Nevertheless, we suppose the avalanche size distribution for a given danger level to be fairly robust and rather independent of terrain and climate characteristics. However, the frequency and intensity of avalanche occurrence will certainly vary between regions.

The proposed size classification based on perimeter data had previously been used to study indicator avalanches in the regions of Davos (Schweizer et al., 2012). With the suggested classification criteria, the resulting median length (Table 1) is well in line with the typical values associated with the corresponding size classes provided by the European Avalanche Warning Services (EAWS, 2019b). They indicate typical length categories of $<50 \mathrm{~m}, 50-200 \mathrm{~m}$, several hundred meters and $1-2 \mathrm{~km}$ for size classes 1 to 4 , respectively.

We then compared avalanche activity to the forecast danger level. Again, this is far from perfect as one would need the verified danger level to compare with. Whereas verification at the lower danger levels can be done with numerous snow instability tests (Schweizer et al., 2003), with measurements (Reuter et al., 2015) or by expert opinion (Techel and Schweizer, 2017), at the higher danger levels (4-High and 5-Very High) avalanche activity is the most reliable hazard indicator.

As no consistent verification data existed for the entire data set, we had to use the forecast. Still, we tried to remove some obvious outliers such as days with forecast danger level 4-High but no avalanche records. This quality check should not be considered comprehensive verification. In total, we changed fewer than $4 \%$ of the danger ratings and mostly increased the danger level (Table S1). In contrast, most verification studies showed a forecast accuracy of about $70 \%-80 \%$ and a trend to over-forecasting (e.g., Techel and
Schweizer, 2017). Hence, our avalanche danger data are still biased, yet also reflect some past and recent practice of applying the danger levels. For example, the danger level 4High was relatively rarely forecast (on fewer than $3 \%$ of the days). This may partly be explained by the location of Davos, which is somewhat protected from major storms, but also relates to forecast practice in Switzerland (Techel et al., 2018). However, it is also remarkable that similar avalanche activity was often differently rated for dry-snow and wet-snow conditions - at all danger levels. This finding adds to the list of inconsistencies in avalanche warning as recently reported in several studies (Clark and Haegeli, 2018; Lazar et al., 2016; Techel et al., 2018).

Correcting the forecast danger level based on avalanche activity, while analyzing the relation between danger level and avalanche activity, may seem questionable. The main effect of the correction procedure was that the median AAI for days with level 4-High increased from about 10 to about 21 . For days with level 3-Considerable, the median AAI was 1 and did not change due to the corrections. Hence, the difference in avalanche activity between levels 3-Considerable and 4-High was already very prominent before the correction procedure (before and after the corrections: $U$ test, $p<0.001)$. With regard to avalanche size, the effects are less prominent. Size 2 avalanches were the most frequent ones at danger levels 1-Low to 4-High before and after the corrections.

The avalanche size distribution we found was remarkably robust with regard to different data stratifications. In particular, the size distribution did not change substantially with the danger level (Fig. 3). In other words, for our data set, avalanche size did not prominently increase with increasing danger level, at least for the danger levels 1-Low to 4-High; size 2 avalanches were the most frequent (Fig. 3b). This finding seems somewhat surprising, given that the avalanche danger level is characterized by a combination of the probability of avalanche occurrence and expected avalanche size (Meister, 1995); it suggests that avalanche size may be of secondary importance compared to snowpack stability and its distribution when assessing the danger level (Techel et al., 2020). Hence, it seems unlikely that the typical (or most frequent) avalanche size is decisive for choosing between four different danger levels for one given snow stability scenario as suggested recently by Müller et al. (2016a). Also, in the conceptual model of avalanche hazard (CMAH) a frequency-magnitude (or likelihood-size) matrix was suggested to estimate avalanche hazard (Statham et al., 2018).

On the other hand, considering the largest avalanche recorded on a given day as suggested by Techel et al. (2020) showed more prominent differences between the danger levels 1-Low to 2-Moderate compared to levels 4High to 5-Very High (Fig. 3d). Hence, the maximum expected avalanche size may be useful to differentiate between some hazard situations (Techel et al., 2020). Clark and 
Haegeli (2018) also reported maximum expected size to be the second most relevant factor for selecting a hazard rating.

Moreover, our findings on avalanche size are in line with the results of a study on avalanche incidents in relation to the danger rating. Harvey (2002) reported that length, width and fracture depth of human-triggered avalanches were very similar at the danger levels 1-Low to 4-High and hence did not increase with increasing danger level; the median length was 200 to $250 \mathrm{~m}$ and the width 50 to $60 \mathrm{~m}$, which corresponds to avalanche size 2, in agreement with our analysis. However, when Harvey (2002) considered all avalanches that had caused damage, i.e., not only human-triggered avalanches but also avalanches that had destroyed trees or infrastructure, he found avalanche size to be larger at the danger levels 4-High and 5-Very High than at the lower danger levels. Logan and Greene (2018) also recently related avalanche size to danger level. They reported that size 2 avalanches (destructive size) were the most frequent size at the danger levels 2-Moderate, 3-Considerable and 4-High - in agreement with our findings.

The relative frequency of the avalanche size classes 2,3 and 4 were $70 \%, 23 \%$ and $2 \%$, respectively. Hence the frequency of avalanche sizes 2 to 4 decreased with increasing size (Table 3, bottom row). Stoffel et al. (1998) also reported decreasing frequency of occurrence for their avalanche size classes medium, large and very large. These findings are in line with the magnitude-frequency relation of most natural hazard events including earthquakes for which the relation is known as the Gutenberg-Richter law (Jentsch et al., 2006). Several other studies have shown frequency-size power laws for snow avalanches (e.g., Birkeland and Landry, 2002; Faillettaz et al., 2004). The fact that size 1 avalanches were not the most frequent, as theoretically should be the case, is probably related to an observation bias: small avalanches may often not be mapped, in particular when other larger avalanches occur, or cannot be mapped at all if they are too small. In fact, when considering avalanche length and plotting a cumulative curve of events, avalanches shorter than $100 \mathrm{~m}$ seem to be underrepresented. This type of observation bias is also present in earthquake catalogues (e.g., Woessner and Wiemer, 2005).

The average number of observed natural avalanches strongly increased with increasing danger level: 2.1, 2.5, 6 and 48 for the danger levels 1-Low to 4-High, respectively. This suggests that the differentiation between the lower two danger levels cannot be based on avalanche occurrence. Also, the relative increase from one danger level to the other is increasing - suggesting an exponential increase in the hazard. Previously, it was suggested that the hazard would double from one level to the other (Munter, 2003). Using accident data a 2- to 3-fold increase was shown (Pfeifer, 2009; Techel et al., 2015), whereas a survey among North American avalanche professionals suggested a 10-fold increase in triggering probability when the regional danger increases by one level (Jamieson et al., 2009b). Based on avalanche observations from Colorado, Elder and Armstrong (1987) assigned avalanche frequencies per day to the four danger levels that were in use at those times: 0-3, 4-9, 10-20 and $\geq 21$.

The prominent, nonlinear increase in avalanche occurrence with increasing danger level reflects that, according to its definition, the avalanche danger level increases with decreasing snow stability. With decreasing snow stability, the frequency of locations with a potential weakness where an avalanche may be released increases. However, the suggested increase in weaknesses in the snowpack can only be assessed with spatial variability studies (e.g., Reuter et al., 2016), which are most appropriate to determine the spatial distribution of instabilities. For example, Schweizer et al. (2003) reported an increase in the proportion of poorly rated profiles from virtually $0 \%$ to $24 \%$ to $53 \%$ for the danger levels 1-Low to 3-Considerable, respectively. Their observations correspond to our finding that the number of natural dry-snow avalanches doubled from level 2-Moderate to 3-Considerable and increased by almost a factor of 3 for wet-snow avalanches. Whereas the number of natural dry-snow avalanches consistently increased with increasing danger level, this was not the case for the human-triggered avalanches. The frequency of human-triggered avalanches did not increase from level 3-Considerable to 4-High. This finding does not mean that triggering becomes less likely but rather reflects terrain usage and the effect of avalanche warnings. In fact, Techel et al. (2015) showed a decrease in ski touring activity already from level 2-Moderate to 3-Considerable and even more prominently from level 3Considerable to 4-High. On the other hand, Wäger and Zweifel (2008) found no decrease in frequency usage from levels 2-Moderate to 3-Considerable when considering offpiste skiing.

At danger level 4-High, the average number of natural avalanches per day in our study region was about 20 for drysnow avalanches and about 50 for wet-snow avalanches. If we assume that "many" natural avalanches are typically observed at the danger level 4-High (EAWS, 2019a), we may conclude that $10-20$ avalanches per $100 \mathrm{~km}^{2}$ have to be expected, considering some underreporting in our study area of about $300 \mathrm{~km}^{2}$, where about two-thirds is avalanche terrain. Hence, our analyses suggest that in the terrain typical of our study area at least about 10 natural avalanches have to be observed for verifying the danger level 4-High. Based on satellite images, Bründl et al. (2019) recently analyzed the avalanche activity during a major avalanche cycle in January 2018 when the danger level was 5-Very High. They analyzed the number of size 4 avalanches per area; for an area of $250 \mathrm{~km}^{2}$ the number of size 4 avalanches ranged from fewer than 29 to up to 202 - roughly consistent with our observations.

With regard to the definition of the avalanche danger by snow stability, its spatial distribution and avalanche size, we would like to point out that the spatial distribution does only refer to the frequency of locations with very poor snow stability where avalanches can initiate from. In other words, where 
the points with very poor snow stability are located in space is irrelevant; only their frequency counts when deciding on a given danger level. Moreover, there is a difference between the probability of avalanche release, which is a local property related to local snow instability as recently revisited by Reuter and Schweizer (2018), and the avalanche occurrence probability, which depends on scale and is the result of stability and its distribution (frequency of triggering spots) for a given area. Moreover, there is a third probability, not to be confused with the two previously mentioned, namely the probability of triggering an avalanche faced by an individual who travels in avalanche terrain on a given day with a given danger level, which also depends on scale (terrain traveled) and terrain choices.

Finally, when the definition for level 2-Moderate danger states that "large natural avalanches are unlikely" (EAWS, 2019a), this definition could also be modified to "natural avalanches are unlikely" since the probability for any size of natural avalanche at level 2-Moderate is less than $5 \%$, which according to the IPCC (IPCC, 2014) is best described by "very unlikely". Likewise, the formulation in the definition of level 1-Low, "only small and medium avalanches are possible", is not appropriate and should be modified. Hence, our analyses of the avalanche activity may be used to improve the descriptions in the avalanche danger scale.

\section{Conclusions}

We quantified some of the key characteristics such as frequency and size of avalanches at a given danger level. To this end, we analyzed a unique data set of 21 years of visually observed avalanche records from the surroundings of Davos (eastern Swiss Alps, $300 \mathrm{~km}^{2}$ ), consisting of the mapped outlines of 13745 avalanches, and we compared the characteristics of avalanche activity to the regional danger level as forecast on $3533 \mathrm{~d}$.

The proportion of days with natural avalanches at a given danger level substantially increased with increasing danger level. Also, the overall number of avalanches per day prominently increased, which reflects the fact that with increasing danger level snow stability decreases and the frequency of locations with a potentially critical weakness in the snowpack increases. The average recorded number of avalanches per day in our study region $\left(300 \mathrm{~km}^{2}\right)$ was $2,3,8$ and 64 for the danger levels 1-Low to 4-High, respectively.

The relative frequency of the four avalanche size classes did not substantially change with increasing danger level, either for human-triggered or for natural avalanches, except for danger level 5-Very High. In other words, avalanche size did not increase with increasing danger level: the most frequent avalanches were size 2 avalanches at any danger level. This suggests that avalanche size may be of secondary importance compared to snowpack stability and its distribution when assessing the danger level. Only in certain situations might avalanche size be decisive - and rather by considering the maximum expected size. Still, the absolute number of very large avalanches (size 4) per day prominently increased, namely by a factor of 20 from level 3-Considerable to 4-High.

At a given danger level the frequency of natural avalanches was typically larger for wet-snow conditions than for drysnow conditions, and wet-snow avalanches tended to be larger - potentially reflecting inconsistent usage of the danger scale in Switzerland. Based on our findings, we propose revisiting the definitions of the danger scale and possibly quantifying some of the descriptions. For example, we suggest that "many avalanches" may mean on the order of at least about 10 avalanches per $100 \mathrm{~km}^{2}$.

We are aware that visual observations are notoriously incomplete. Hence, our results should be challenged by similar analyses with similarly extensive data sets. In the future, more comprehensive data sets based on remotely sensed data and results from avalanche detection systems may allow better founded analyses.

Finally, our analyses confirm that different avalanche situations are typically condensed into one specific danger level, which results in a loss of information. Hence, avalanche warning services are encouraged to describe the danger as best as they can and not only provide the danger level. Likewise, risk assessment in avalanche education should not focus solely on the danger level, or the release probability, but also include the potential consequences.

Data availability. The data sets of avalanche observations and avalanche danger are accessible at https://doi.org/10.16904/envidat.134 (Schweizer et al., 2020).

Supplement. The supplement related to this article is available online at: https://doi.org/10.5194/tc-14-737-2020-supplement.

Author contributions. JS, CM and BR designed the study. AS, FT, JS and CM extracted and curated the data. JS analyzed the data and prepared the manuscript with contributions from all co-authors.

Competing interests. The authors declare that they have no conflict of interest.

Acknowledgements. We would like to thank everyone who contributed to the avalanche occurrence data set, in particular the interns of the avalanche warning service at SLF, who mapped most of the avalanches. We are grateful for the constructive comments by the two reviewers and the editor that helped to improve the paper. 
Review statement. This paper was edited by Evgeny A. Podolskiy and reviewed by Rune Engeset and Pascal Haegeli.

\section{References}

Birkeland, K. and Landry, C. C.: Power-laws and snow avalanches, Geophys. Res. Lett., 29, 1554, https://doi.org/10.1029/2001GL014623, 2002.

Bründl, M., Hafner, E., Bebi, P., Bühler, Y., Margreth, S., Marty, C., Schaer, M., Stoffel, L., Techel, F., Winkler, K., Zweifel, B., and Schweizer, J.: Ereignisanalyse Lawinensituation im Januar 2018, WSL Bericht, 76, edited by: Steffen, K., Federal Research Institute WSL, Birmensdorf, Switzerland, 162 pp., 2019.

Clark, T.: Exploring the link between the conceptual model of avalanche hazard and the North American public avalanche danger scale, M.Sc., Faculty of Environement, Simon Fraser University, Burnaby BC, Canada, 104 pp., 2019.

Clark, T. and Haegeli, P.: Establishing the link between the conceptual model of avalanche hazard and the North American public avalanche danger scale: initial explorations from Canada, Proceedings ISSW 2018, International Snow Science Workshop, Innsbruck, Austria, 7-12 October 2018, 1116-1120, 2018.

Dennis, A. and Moore, M.: Evolution of public avalanche information: The north American experience with avalanche danger rating levels, Proceedings ISSW 1996, International Snow Science Workshop, Banff, Alberta, Canada, 6-10 October 1996, 60-66, 1997.

EAWS: Avalanche Danger Scale, available at: https://www. avalanches.org/standards/avalanche-danger-scale/, last access: 27 July 2019a.

EAWS: Avalanche Size, available at: https://www.avalanches.org/ standards/avalanche-size/, last access: 27 July 2019b.

EAWS: EAWS Matrix, available at: https://www.avalanches.org/ standards/eaws-matrix/, last access: 17 January 2020.

Eckerstorfer, M., Malnes, E., and Müller, K.: A complete snow avalanche activity record from a Norwegian forecasting region using Sentinel-1 satellite-radar data, Cold Reg. Sci. Technol., 144, 39-51, https://doi.org/10.1016/j.coldregions.2017.08.004, 2017.

Eckert, N., Parent, E., Kies, R., and Baya, H.: A spatio-temporal modelling framework for assessing the fluctuations of avalanche occurrence resulting from climate change: application to 60 years of data in the northern French Alps, Clim. Change, 101, 515-553, https://doi.org/10.1007/s10584-009-9718-8, 2010.

Elder, K. and Armstrong, B.: A quantitative approach for verifying avalanche hazard ratings, Symposium at Davos 1986 Avalanche Formation, Movement and Effects, IAHS Publ., 162, 593-601, 1987.

Faillettaz, J., Louchet, F., and Grasso, J. R.: Twothreshold model for scaling laws of noninteracting snow avalanches, Phys. Rev. Lett., 93, 208001/208001-208004, https://doi.org/10.1103/PhysRevLett.93.208001, 2004.

Föhn, P. M. B. and Schweizer, J.: Verification of avalanche danger with respect to avalanche forecasting, in: Les apports de la recherche scientifique à la sécurite neige, glace et avalanche. Actes de Colloque, Chamonix, 30 May-3 June 1995, edited by: Sivardière, F., ANENA, Grenoble, France, 151-156, 1995.
Haegeli, P., Falk, M., and Klassen, K.: Linking avalanche problems to avalanche danger - A first statistical examination of the conceptual model of avalanche hazard, Proceedings ISSW 2012, International Snow Science Workshop Anchorage AK, USA, 1621 September 2012, 859-865, 2012.

Harvey, S.: Avalanche incidents in Switzerland in relation to the predicted danger degree, Proceedings ISSW 2002, International Snow Science Workshop, Penticton BC, Canada, 29 September4 October 2002, 443-448, 2002.

Harvey, S., Schmudlach, G., Bühler, Y., Dürr, L., Stoffel, A., and Christen, M.: Avalanche terrain maps for backcountry skiing in Switzerland, Proceedings ISSW 2018, International Snow Science Workshop, Innsbruck, Austria, 7-12 October 2018, 16251631, 2018.

Hendrikx, J., Owens, I., Carran, W., and Carran, A.: Avalanche activity in an extreme maritime climate: The application of classification trees for forecasting, Cold Reg. Sci. Technol., 43, 104116, https://doi.org/10.1016/j.coldregions.2005.05.006, 2005.

IPCC: Climate Change 2014: Synthesis Report. Contribution of Working Groups I, II and III to the Fifth Assessment Report of the Intergovernmental Panel on Climate Change, IPCC, Geneva, Switzerland, 151 pp., 2014.

Jamieson, B., Haegeli, P., and Schweizer, J.: Field observations for estimating the local avalanche danger in the Columbia Mountains of Canada, Cold Reg. Sci. Technol., 58, 84-91, https://doi.org/10.1016/j.coldregions.2009.03.005, 2009a.

Jamieson, B., Schweizer, J., and Shea, C.: Simple calculations of avalanche risk for backcountry skiing, Proceedings ISSW 2009, International Snow Science Workshop, Davos, Switzerland, 27 September-2 October 2009, 336-340, 2009b.

Jentsch, V., Kantz, H., and Albeverio, S.: Extreme events: magic, mysteries and challenges, in: Extreme events in nature and society, edited by: Jentsch, V., Kantz, H., and Albeverio, S., Springer, Berlin, Germany, 1-18, 2006.

Lacroix, P., Grasso, J. R., Roulle, J., Giraud, G., Goetz, D., Morin, S., and Helmstetter, A.: Monitoring of snow avalanches using a seismic array: Location, speed estimation, and relationships to meteorological variables, J. Geophys. Res.-Earth Surf., 117, F01034, https://doi.org/10.1029/2011jf002106, 2012.

Lazar, B., Trautman, S., Cooperstein, M., Greene, E., and Birkeland, K.: North American avalanche danger scale: are public backcountry forecasters applying it consistently?, Proceedings ISSW 2016, International Snow Science Workshop, Breckenridge CO, USA, 3-7 October 2016, 457-465, 2016.

Logan, S. and Greene, E.: Patterns in avalanche events and regional scale avalanche forecasts in Colorado, USA, Proceedings ISSW 2018, International Snow Science Workshop, Innsbruck, Austria, 7-12 October 2018, 1059-1062, 2018.

McClung, D. M.: The elements of applied avalanche forecasting - Part I: The human issues, Nat. Hazards, 26, 111-129, https://doi.org/10.1023/a:1015665432221, 2002.

McClung, D. M. and Schaerer, P.: Snow avalanche size classification, Proceedings of Avalanche Workshop, Vancouver BC, Canada, 3-5 November 1980, 12-27, 1981.

McClung, D. M. and Schaerer, P.: The Avalanche Handbook, 3rd ed., The Mountaineers Books, Seattle WA, USA, 342 pp., 2006.

Meister, R.: Country-wide avalanche warning in Switzerland, Proceedings International Snow Science Workshop, Snowbird, Utah, USA, 30 October-3 November 1994, 1995. 
Müller, K., Mitterer, C., Engeset, R. V., Ekker, R., and Kosberg, S. Ø.: Combining the conceptual model of avalanche hazard with the Bavarian matrix, Proceedings ISSW 2016, International Snow Science Workshop, Breckenridge CO, USA, 3-7 October 2016, 472-479, 2016a.

Müller, K., Stucki, T., Mitterer, C., Nairz, P., Konetschny, H., Feistl, T., Coléou, C., Berbenni, G., and Chiambretti, I.: Towards an improved European auxiliary matrix for assessing avalanche danger levels, Proceedings ISSW 2016, International Snow Science Workshop, Breckenridge CO, USA, 3-7 October 2016, 12291231, 2016b.

Munter, W.: $3 \times 3$ Lawinen - Risikomanagement im Wintersport, Pohl\&Schellhammer, Garmisch-Partenkirchen, Germany, 223 pp., 2003.

Pfeifer, C.: On probabilities of avalanches triggered by alpine skiers. An empirically driven decision strategy for backcountry skiers based on these probabilities, Nat. Hazards, 48, 425-438, https://doi.org/10.1007/s11069-008-9270-2, 2009.

Reuter, B. and Schweizer, J.: Describing snow instability by failure initiation, crack propagation, and slab tensile support, Geophys. Res. Lett., 45, 7019-7027, https://doi.org/10.1029/2018GL078069, 2018.

Reuter, B., van Herwijnen, A., Veitinger, J., and Schweizer, J.: Relating simple drivers to snow instability, Cold Reg. Sci. Technol., 120, 168-178, https://doi.org/10.1016/j.coldregions.2015.06.016, 2015.

Reuter, B., Richter, B., and Schweizer, J.: Snow instability patterns at the scale of a small basin, J. Geophys. Res.-Earth Surf., 121, 257-282, https://doi.org/10.1002/2015JF003700, 2016.

Schweizer, J.: On the predictability of snow avalanches, Proceedings ISSW 2008, International Snow Science Workshop, Whistler BC, Canada, 21-27 September 2008, 688-692, 2008.

Schweizer, J.: Predicting the avalanche danger level from field observations, Proceedings ISSW 2010, International Snow Science Workshop, Lake Tahoe CA, USA, 17-22 October 2010, 162$165,2010$.

Schweizer, J., Kronholm, K., and Wiesinger, T.: Verification of regional snowpack stability and avalanche danger, Cold Reg. Sci. Technol., 37, 277-288, https://doi.org/10.1016/S0165232X(03)00070-3, 2003.

Schweizer, J., Alig, C., and Mitterer, C.: On indicator path avalanches for local avalanche forecasting, Proceedings ISSW 2012, International Snow Science Workshop, Anchorage AK, U.S.A., 16-21 September 2012, 51-54, 2012.

Schweizer, J., Mitterer, C., Techel, F., Stoffel, A., and Reuter, B.: Snow avalanche data Davos, Switzerland, 1999-2019, EnviDat, https://doi.org/10.16904/envidat.134, 2020.

Silver, N.: The Signal and the Noise - The Art and Science of Prediction, Pinguin Books, 544 pp., 2012.
Spiegel, M. R. and Stephens, L. J.: Schaum's Outline of Theory and Problems of Statistics, 3rd ed., Schaum's outline series, McGraw-Hill, New York, 538 pp., 1999.

Statham, G., Haegeli, P., Birkeland, K. W., Greene, E., Israelson, C., Tremper, B., Stethem, C., McMahon, B., White, B., and Kelly, J.: The North American public avalanche danger scale, Proceedings ISSW 2010, International Snow Science Workshop, Lake Tahoe CA, USA, 17-22 October 2010, 117-123, 2010.

Statham, G., Haegeli, P., Greene, E., Birkeland, K., Israelson, C., Tremper, B., Stethem, C., McMahon, B., White, B., and Kelly, J.: A conceptual model of avalanche hazard, Nat. Hazards, 90, 663-691, https://doi.org/10.1007/s11069-017-3070-5, 2018.

Stoffel, A., Meister, R., and Schweizer, J.: Spatial characteristics of avalanche activity in an alpine valley - a GIS approach, Ann. Glaciol., 26, 329-336, https://doi.org/10.3189/1998AoG26-1329-336, 1998.

Techel, F. and Schweizer, J.: On using local avalanche danger level estimates for regional forecast verification, Cold Reg. Sci. Technol., 144, 52-62, https://doi.org/10.1016/j.coldregions.2017.07.012, 2017.

Techel, F., Zweifel, B., and Winkler, K.: Analysis of avalanche risk factors in backcountry terrain based on usage frequency and accident data in Switzerland, Nat. Hazards Earth Syst. Sci., 15, 19851997, https://doi.org/10.5194/nhess-15-1985-2015, 2015.

Techel, F., Mitterer, C., Ceaglio, E., Coléou, C., Morin, S., Rastelli, F., and Purves, R. S.: Spatial consistency and bias in avalanche forecasts - a case study in the European Alps, Nat. Hazards Earth Syst. Sci., 18, 2697-2716, https://doi.org/10.5194/nhess18-2697-2018, 2018.

Techel, F., Müller, K., and Schweizer, J.: On the importance of snowpack stability, its frequency distribution, and avalanche size in assessing the avalanche danger level: a data-driven approach, The Cryosphere Discuss., https://doi.org/10.5194/tc-2020-42, in review, 2020.

Ulivieri, G., Marchetti, E., Ripepe, M., Chiambretti, I., Rosa, G. D., and Segor, V.: Monitoring snow avalanches in Northwestern Italian Alps using an infrasound array, Cold Reg. Sci. Technol., 69, 177-183, https://doi.org/10.1016/j.coldregions.2011.09.006, 2011.

van Herwijnen, A. and Schweizer, J.: Monitoring avalanche activity using a seismic sensor, Cold Reg. Sci. Technol., 69, 165-176, https://doi.org/10.1016/j.coldregions.2011.06.008, 2011.

Wäger, P. and Zweifel, B.: 10hochminus5, Bergundsteigen Zeitschrift für Risikomanagement im Bergsport, Oesterreichischer Alpenverein, Innsbruck, Austria, 17, 22-36, 2008.

Woessner, J. and Wiemer, S.: Assessing the quality of earthquake catalogues: estimating the magnitude of completeness and its uncertainty, B. Seismol. Soc. Am., 95, 684-698, https://doi.org/10.1785/0120040007, 2005. 